\title{
Uniform Exponential Stability of Perturbed Semigroups: The Dyson-Phillips Formula Versus Gil's Approach Via Commutators
}

\author{
Adam Gregosiewicz@
}

\begin{abstract}
We discuss the uniform exponential stability of strongly continuous semigroups generated by operators of the form $A+B$, where $B$ is a bounded perturbation of a generator $A$. We compare two approaches to the problem: via the Dyson-Phillips formula and via the size of the norm of the commutator of $A$ and $B$ - the method recently developed by M. Gil'. We show that quite often the first approach is more powerful than the second one and, more importantly, easier to use.
\end{abstract}

Mathematics Subject Classification. 47D06, 47D60.

Keywords. Semigroups of operators, uniform exponential stability, perturbations, commutator.

\section{Introduction}

We say that a strongly continuous semigroup of operators $\left\{\mathrm{e}^{t A}\right\}_{t>0}$, generated by an operator $A$ in a Banach space $X$, is uniformly exponentially stable if the growth bound

$$
\omega_{0}:=\inf \left\{\omega \in \mathbb{R}:\left\|\mathrm{e}^{t A}\right\| \leq M_{\omega} \mathrm{e}^{\omega t} \text { for some } M_{\omega} \geq 1 \text { and all } t \geq 0\right\}
$$

of the semigroup is negative. There are several conditions equivalent to $\omega_{0}<0$, see [5, V.1.7]. However, in the context of the paper, we note that, by the Datko and Pazy theorem [5, V.1.8], the semigroup generated by $A$ is uniformly exponentially stable if and only if the map $t \mapsto\left\|\mathrm{e}^{t A}\right\|$ is integrable over $(0,+\infty)$, 
that is if and only if

$$
\int_{0}^{+\infty}\left\|\mathrm{e}^{t A}\right\| \mathrm{d} t<+\infty .
$$

We concentrate on the uniform exponential stability of perturbed semigroups. Given a generator $A$ and a bounded operator $B$ in $X$, by the Bounded Perturbation Theorem [5, III.1.3] it follows that the operator $A+B$ generates a strongly continuous semigroup, and we can ask under what conditions the semigroup is uniformly exponentially stable. The problem is well known and discussed by many authors - see for example Preda's survey [10] and references given there.

A direct way to approach the question of stability of perturbed semigroups is via the Dyson-Phillips formula [5, III.1.10], which we discuss in Sect. 3. On the other hand, the classical Liapunov Stability Theorem [5, I.2.10], which states that the semigroup generated by a bounded operator (matrix) $A$ in $\mathbb{C}^{n}$ is uniformly exponentially stable if and only if the real parts of all eigenvalues of $A$ are negative, suggests that an analysis of the spectrum of a generator may be helpful. Indeed, see [5, V.1.10], if a semigroup is eventually norm-continuous, then it is uniformly exponentially stable if and only if the spectral bound

$$
\mathrm{s}(A):=\sup \{\operatorname{Re} \lambda: \lambda \text { belongs to the spectrum } \sigma(A)\}
$$

of its generator $A$ is negative. This idea can be carried over to perturbed semigroups. It turns out, see [4] and [10], that if at least one element of the Dyson-Phillips series, see (3.1), is norm-continuous, then the semigroup generated by a bounded perturbation $A+B$ of $A$ is uniformly exponentially stable if and only if the spectral bound $s(A+B)$ is negative. In Hilbert spaces there is also a resolvent related condition that is equivalent to the uniform exponential stability, see the Gearhart-Prüss-Greiner theorem [5, V.1.11].

Recently, Gil' $[6,7]$ found yet another approach to the uniform exponential stability of perturbed semigroups via the commutator of generators $A$ and $B$. More precisely, he proved that for a generator $A$ and a bounded operator $B$ in a Banach space $X$, the semigroup generated by $A+B$ is uniformly exponentially stable, provided that the following conditions hold:

(a) $\mathcal{J}:=\int_{0}^{+\infty}\left\|\mathrm{e}^{t A}\right\|\left\|\mathrm{e}^{t B}\right\| \mathrm{d} t<+\infty$,

(b) $\mathcal{D}(A)$ is invariant for $B$, that is $B(\mathcal{D}(A)) \subset \mathcal{D}(A)$, and $A B-B A$ has a bounded extension $K$ to $X$,

(c) $\|K\| \mathcal{J}^{2}<1$.

The result was slightly improved by A. Bobrowski, who showed in [3] that the conclusion of Gil's theorem still holds if we replace (c) with a weaker condition

(d) $\|K\| \mathcal{I}<1$ 
where $\mathcal{I}:=\int_{0}^{+\infty}\left\|\mathrm{e}^{t B}\right\| \int_{0}^{t}\left\|\mathrm{e}^{(t-s) A}\right\|\left\|\mathrm{e}^{s A}\right\| \mathrm{d} s \mathrm{~d} t$. Moreover, he provided an example of a uniformly exponentially stable perturbed semigroup such that conditions (a)-(b) hold, while the norm of $K$ is arbitrarily large and condition (c) or (d) fails. This suggests that the size of the commutator of generators is not the best criterion for the stability of perturbed semigroups.

We try to understand how Gil's method relates to the approach via the Dyson-Phillips formula. First we show, see Sect. 2, that neither condition (a) nor (b) is necessary for the uniform exponential stability of perturbed semigroups, and, on the other hand, that there are (even bounded) generators $A$ and $B$ satisfying (a)-(b), such that the semigroup generated by $A+B$ is not uniformly exponentially stable. This means that we cannot omit condition (c) or (d) in Gil's theorem, and that the size of the norm of $K$ plays a role here. Next, in Sect. 3, we state a simple corollary, see Proposition 3.1, of the Dyson-Phillips theorem, that is useful for proving uniform exponential stability of perturbed semigroups. In particular, for one example considered by Gil' in [6], we give a simpler and simultaneously better estimate on when the discussed semigroup, depending on a parameter, is uniformly exponentially stable. In Sect. 4 we provide a class of examples of bounded perturbations of a semigroup resembling the translation semigroup in $L^{1}$-type space, and compare in this case both approaches to the uniform exponential stability - via the Dyson-Phillips formula and via Gil's estimate of the norm of the commutator. Finally, in Sect. 5, we show that the examples discussed in Sect. 4 fall into a general scheme in an abstract Banach space. We prove that in this scheme the Dyson-Phillips series gives an explicit formula for the perturbed semigroup, which allows us to provide an efficient condition for its uniform exponential stability. As far as we understand, in many situations the approach via the Dyson-Phillips formula is at least as powerful as that of Gil'; see, however, Remark 4.3. What is even more important, the first one is easier to use.

\section{Discussion of Gil's Conditions}

First we notice that condition (b) is not necessary for the uniform exponential stability of a perturbed semigroup. Indeed, if $A+B$ is a generator of a strongly continuous semigroup in a Banach space $X$, then $\left\|\mathrm{e}^{t(A+B)}\right\| \leq M \mathrm{e}^{\omega t}$ for some $M \geq 1, \omega \in \mathbb{R}$, and all $t \geq 0$. Hence,

$$
\left\|\mathrm{e}^{t(A+B-(1+\omega) I)}\right\|=\left\|\mathrm{e}^{-(1+\omega) t} \mathrm{e}^{t(A+B)}\right\| \leq M \mathrm{e}^{-t}, \quad t \geq 0,
$$

where $I$ is the identity operator in $X$, which implies that the semigroup generated by

$$
A+B_{\omega}:=A+[B-(1+\omega) I]
$$

is uniformly exponentially stable, no matter what $A$ and $B$ are.

Next we show that condition (a) is not necessary either. 
Example 2.1. Consider the space $\mathbb{R}^{2}$ equipped with the norm $\|(x, y)\|_{1}=|x|+$ $|y|$. Let operators $A$ and $B$ in $\mathbb{R}^{2}$ be defined by matrices

$$
A=\left[\begin{array}{cc}
-2 & 1 \\
0 & 0
\end{array}\right], \quad B=\left[\begin{array}{cc}
0 & 0 \\
1 & -2
\end{array}\right] \text {. }
$$

By $[5$, I.2.7] the related semigroups are given by

$$
\mathrm{e}^{t A}=\left[\begin{array}{cc}
\mathrm{e}^{-2 t} & \mathrm{e}^{-t} \sinh t \\
0 & 1
\end{array}\right], \quad \mathrm{e}^{t B}=\left[\begin{array}{cc}
1 & 0 \\
\mathrm{e}^{-t} \sinh t \mathrm{e}^{-2 t}
\end{array}\right]
$$

for all $t \geq 0$. Hence, the operator norms $\left\|\mathrm{e}^{t A}\right\|_{1}$ and $\left\|\mathrm{e}^{t B}\right\|_{1}$ converge to $3 / 2$ as $t \rightarrow+\infty$, and consequently $\int_{0}^{+\infty}\left\|\mathrm{e}^{t A}\right\|_{1}\left\|\mathrm{e}^{t B}\right\|_{1} \mathrm{~d} t$ diverges, which means that condition (a) does not hold here. On the other hand, again by [5, I.2.7],

$$
\mathrm{e}^{t(A+B)}=\mathrm{e}^{-2 t}\left[\begin{array}{l}
\cosh t \sinh t \\
\sinh t \cosh t
\end{array}\right], \quad t \geq 0,
$$

and clearly the semigroup generated by $A+B$ is uniformly exponentially stable, since $\left\|\mathrm{e}^{t(A+B)}\right\|_{1}$ is of order $\mathrm{e}^{-t}$ as $t \rightarrow+\infty$.

As we indicated in Introduction there exist, see [3], uniformly exponentially stable semigroups generated by $A+B$ with arbitrarily large norm of the commutator $K$ of $A$ and $B$. This naturally leads to the question if conditions (a)-(b), without (c) or (d), are sufficient for the uniform exponential stability of $\left\{\mathrm{e}^{t(A+B)}\right\}_{t>0}$. In the following example we show that the answer is in the negative, and there are bounded operators $A$ and $B$ satisfying conditions (a)-(b), such that the semigroup generated by $A+B$ is not uniformly exponentially stable. In other words, this means that the size of the norm of $K$ plays a role in the discussed problem.

Example 2.2. As in Example 2.1 we consider $\mathbb{R}^{2}$ with the same norm, and define

$$
A=\left[\begin{array}{cc}
-1 & 3 \\
0 & -1
\end{array}\right], \quad B=A^{*}
$$

where $A^{*}$ is the transpose of $A$. Using $[5$, I.2.7] we calculate

$$
\mathrm{e}^{t A}=\left(\mathrm{e}^{t B}\right)^{*}=\mathrm{e}^{-t}\left[\begin{array}{cc}
1 & 3 t \\
0 & 1
\end{array}\right], \quad t \geq 0 .
$$

It follows that conditions (a)-(b) are satisfied, however,

$$
\mathrm{e}^{t(A+B)}=\mathrm{e}^{-2 t}\left[\begin{array}{c}
\cosh 3 t \\
\sinh 3 t \\
\sinh 3 t \cosh 3 t
\end{array}\right], \quad t \geq 0,
$$

which shows that the semigroup generated by $A+B$ is not uniformly exponentially stable, since $\left\|\mathrm{e}^{t(A+B)}\right\|_{1}$ is of order $\mathrm{e}^{t}$.

Note that in this case

$$
\mathcal{J}:=\int_{0}^{+\infty}\left\|\mathrm{e}^{t A}\right\|_{1}\left\|\mathrm{e}^{t B}\right\|_{1} \mathrm{~d} t=\int_{0}^{+\infty} \mathrm{e}^{-2 t}(1+3 t)^{2} \mathrm{~d} t=\frac{17}{4},
$$


and

$$
K:=A B-B A=\left[\begin{array}{cc}
9 & 0 \\
0 & -9
\end{array}\right] .
$$

Consequently $\|K\|_{1}=9$, and $\|K\|_{1} \mathcal{J}^{2}>1$, which shows directly that condition (c) does not hold.

\section{Dyson-Phillips Formula}

Let $A$ be a generator of a strongly continuous semigroup in a Banach space $X$. If $B$ is a bounded operator in $X$, then the Dyson-Phillips theorem, see [5, III.1.10], says that the semigroup $\left\{\mathrm{e}^{t(A+B)}\right\}_{t \geq 0}$ generated by $A+B$ is given by the series

$$
\mathrm{e}^{t(A+B)}=\sum_{n=0}^{+\infty} T_{n}(t), \quad t \geq 0,
$$

which converges in the operator norm, where $T_{0}(t):=\mathrm{e}^{t A}$ and

$$
T_{n+1}(t):=\int_{0}^{t} \mathrm{e}^{(t-s) A} B T_{n}(s) \mathrm{d} s, \quad n \geq 0
$$

for all $t \geq 0$. If $M \geq 1$ and $\omega \in \mathbb{R}$ are chosen so that

$$
\left\|\mathrm{e}^{t A}\right\| \leq M \mathrm{e}^{\omega t}, \quad t \geq 0
$$

then as a simple consequence of the Dyson-Phillips formula we obtain the estimate

$$
\left\|\mathrm{e}^{t(A+B)}\right\| \leq M \mathrm{e}^{(\omega+M\|B\|) t}, \quad t \geq 0 .
$$

However, if we take a $\mu \in \mathbb{R}$, then $\left\|\mathrm{e}^{t(A-\mu I)}\right\| \leq M \mathrm{e}^{(\omega-\mu) t}$ for all $t \geq 0$, where $I$ is the identity operator in $X$. Consequently

$$
\left\|\mathrm{e}^{t(A+B)}\right\|=\left\|\mathrm{e}^{t[(A-\mu I)+(B+\mu I)]}\right\| \leq M \mathrm{e}^{(\omega-\mu+M\|B+\mu I\|) t}, \quad t \geq 0 .
$$

This leads to the following result.

Proposition 3.1. Let $A$ be a generator of a strongly continuous semigroup in a Banach space $X$, and fix $M \geq 1$ and $\omega \in \mathbb{R}$ such that (3.2) holds. Let $B$ be a bounded operator in $X$. If there exist $\mu \in \mathbb{R}$ such that

$$
\mu-\omega>M\|B+\mu I\|,
$$

then the semigroup generated by $A+B$ is uniformly exponentially stable.

Proof. By (3.4) we have

$$
\left\|\mathrm{e}^{t(A+B)}\right\| \leq M \mathrm{e}^{\nu t}, \quad t \geq 0
$$

for $\nu:=\omega-\mu+M\|B+\mu I\|<0$, which completes the proof. 
We stress that Proposition 3.1 emphasizes the importance of the distance of $B$ to the subspace spanned by the identity operator. What is interesting, in the context of Gil's commutator approach, in every Hilbert space for a given bounded operator $B$ it is possible to find a bounded operator $A$ with $\|A\|=1$ such that the norm of their commutator is arbitrarily close to $\inf _{\mu \in \mathbb{C}} 2\|B+\mu I\|$, see [11, Theorem 4]. This suggests that Gil's conditions and estimate (3.4) are somehow related, at least in Hilbert spaces. However, the nature of this relationship eludes the author.

\subsection{Gil's Example}

As an application of Proposition 3.1 we consider the example discussed by Gil', see Sect. 3, and in particular formula (3.7), in [6].

Let $X=L^{2}\left([0,1], \mathbb{C}^{2}\right)$ be the Hilbert space of $\mathbb{C}^{2}$-valued functions defined on $[0,1]$ with the scalar product

$$
(f, g):=\int_{0}^{1}(f(x), g(x))_{2} \mathrm{~d} x, \quad f, g \in X,
$$

where $(\cdot, \cdot)_{2}$ is the standard scalar product in $\mathbb{C}^{2}$. We introduce the generator $A$ in $X$ by

$$
\text { Af }:=f^{\prime}, \quad f \in \mathcal{D}(A)
$$

with domain

$$
\mathcal{D}(A):=\left\{f \in X: f^{\prime} \in X \text { and } f(0)=f(1)\right\} .
$$

Moreover, for fixed $a \in \mathbb{R}$ we let

$$
M(x):=\left[\begin{array}{cc}
-1 & a \cos \pi x \\
a \sin \pi x, & -1
\end{array}\right], \quad x \in[0,1],
$$

and define the bounded operator $B$ in $X$ by $B f(x):=M(x) f(x), x \in[0,1]$. By applying Gil's result we obtain that the semigroup generated by $A+B$ is uniformly exponentially stable, provided that $a \in(0,1)$ and

$$
a\left[\frac{1}{1-a}+\frac{a}{(1-a)^{2}}\right]^{2}<1,
$$

which holds for all $a \in(0, \alpha)$, where $\alpha$ is approximately 0.28 .

On the other hand, for the identity operator $I$ in $X$ and the $2 \times 2$ identity matrix $I_{2}$, we have

$$
\|B+I\|=\sup _{x \in[0,1]}\left\|M(x)+I_{2}\right\|_{2}
$$

here $\|\cdot\|$ is the operator norm in $X$, and $\|\cdot\|_{2}$ is the operator norm in $\mathbb{C}^{2}$ related to the standard scalar product $(\cdot, \cdot)_{2}$. The norm $\left\|M(x)+I_{2}\right\|_{2}$ is the spectral norm of $M(x)+I_{2}$, see [8, p. 281], which is the square root of the 
largest eigenvalue of $\left(M(x)+I_{2}\right)^{*}\left(M(x)+I_{2}\right)$, where $\left(M(x)+I_{2}\right)^{*}$ is the (conjugate) transpose of $M(x)+I_{2}$. We have

$$
\left(M(x)+I_{2}\right)^{*}\left(M(x)+I_{2}\right)=\left[\begin{array}{cc}
a^{2} \sin ^{2} \pi x & 0 \\
0 & a^{2} \cos ^{2} \pi x
\end{array}\right], \quad x \in[0,1] .
$$

Hence,

$$
\left\|M(x)+I_{2}\right\|_{2}=|a| \max \{\sin \pi x,|\cos \pi x|\}, \quad x \in[0,1],
$$

and consequently $\|B+I\|=|a|$. Since $A$ generates a contraction semigroup, see [6, Sect. 3], we have $\left\|\mathrm{e}^{t(A-I)}\right\| \leq \mathrm{e}^{-t}$ for all $t \geq 0$. Therefore, by Proposition 3.1 with $M=1, \omega=0$, and $\mu=1$, it follows that the semigroup generated by $A+B$ is uniformly exponentially stable, provided that

$$
1>\|B+I\|=|a|
$$

that is for all $a \in(-1,1)$. In comparison to Gil's conclusion, which only gives $a \in(0, \alpha)$, where $\alpha \sim 0.28$, this is a stronger result.

It is even possible to strengthened the result further, by choosing a different shift $\mu$. We show that the semigroup generated by $A+B$ is uniformly exponentially stable, provided that

$$
|a|<\sqrt{2}
$$

Indeed, let $\mu_{a}:=1+|a|$. Then, as before,

$$
\left\|B+\mu_{a} I\right\|=\sup _{x \in[0,1]}\left\|M(x)+\mu_{a} I_{2}\right\|_{2} .
$$

A little bit of algebra shows that the eigenvalues of $\left(M(x)+\mu_{a} I_{2}\right) *(M(x)+$ $\left.\mu_{a} I_{2}\right)$ are given by

$$
\lambda_{ \pm}(x)=a^{2}\left[\frac{3}{2} \pm \frac{1}{2 \sqrt{2}} \sqrt{8(1+\sin (2 \pi x))+1+\cos (4 \pi x)}\right], \quad x \in[0,1] .
$$

Therefore, the supremum of $\max \left\{\lambda_{+}(x), \lambda_{-}(x)\right\}$ over $x \in[0,1]$ is attained at $1 / 4$ and equals

$$
\lambda_{+}(1 / 4)=a^{2}\left(1+\frac{1}{\sqrt{2}}\right)^{2}
$$

which means that $\left\|B+\mu_{a} I\right\|=|a|(1+1 / \sqrt{2})$. Thus, by Proposition 3.1 with $M=1, \omega=0$, and $\mu=\mu_{a}$, we obtain that the semigroup generated by $A+B$ is uniformly exponentially stable, provided that $\mu_{a}>|a|(1+1 / \sqrt{2})$, or, equivalently, $|a|<\sqrt{2}$. 


\section{More Examples}

We provide a class of examples of bounded perturbations of a semigroup resembling the translation semigroup in $L^{1}$-type space. In this setup we compare both approaches to the uniform exponential stability, via Gil's size of the commutator and via the Dyson-Phillips formula. We show, in Sect. 4.1, that even the simplest consequence of the latter, namely the estimate (3.3), is quite powerful and easy to apply.

Let $\mathbb{R}_{+}=[0,+\infty)$ and let $\psi$ be a real-valued locally integrable function on $\mathbb{R}_{+}$satisfying

$$
\sup _{x \in \mathbb{R}_{+}} \int_{x}^{x+1}|\psi(y)| \mathrm{d} y<+\infty .
$$

This guarantees that $\int_{0}^{x} \psi(y) \mathrm{d} y=O(x)$ as $x \rightarrow+\infty$. Denote

$$
\phi(x):=\exp \left(\int_{0}^{x} \psi(y) \mathrm{d} y\right), \quad x \in \mathbb{R}_{+} .
$$

In the space $L^{1}\left(\mathbb{R}_{+}\right)$of Lebesgue integrable functions over $\mathbb{R}_{+}$equipped with the standard norm $\|\cdot\|_{1}$ we introduce the operator $A$ defined by

$$
A f:=f^{\prime}+\psi f=\frac{(\phi f)^{\prime}}{\phi}, \quad f \in \mathcal{D}(A),
$$

with the domain

$$
\mathcal{D}(A)=\left\{f \in L^{1}\left(\mathbb{R}_{+}\right): f \text { is absolutely continuous and }(\phi f)^{\prime} / \phi \in L^{1}\left(\mathbb{R}_{+}\right)\right\} .
$$

We introduce the left translation operator $\tau_{t}$ in $L^{1}\left(\mathbb{R}_{+}\right)$as

$$
\tau_{t} f(x):=f(x+t), \quad t \geq 0, x \in \mathbb{R}_{+}, f \in L^{1}\left(\mathbb{R}_{+}\right),
$$

and denote $\|f\|_{\infty}:=\sup _{x \in \mathbb{R}_{+}}|f(x)|$ for every bounded function $f$ on $\mathbb{R}_{+}$. Note that for $\phi$ defined above we have

$$
M_{t}:=\left\|\frac{\tau_{t} \phi}{\phi}\right\|_{\infty}=\sup _{x \in \mathbb{R}_{+}} \exp \left(\int_{x}^{x+t} \psi(y) \mathrm{d} y\right)<+\infty
$$

for every $t \geq 0$.

Lemma 4.1. The operator $A$ defined by (4.2) generates the strongly continuous semigroup $\left\{\mathrm{e}^{t A}\right\}_{t \geq 0}$ in $L^{1}\left(\mathbb{R}_{+}\right)$given by

$$
\mathrm{e}^{t A} f=\frac{\tau_{t} \phi}{\phi} \tau_{t} f, \quad t \geq 0, f \in L^{1}\left(\mathbb{R}_{+}\right) .
$$

Moreover,

$$
\left\|\mathrm{e}^{t A}\right\|_{1}=M_{t}, \quad t \geq 0 \text {. }
$$

We postpone the proof of Lemma 4.1 to "Appendix". 


\subsection{Bounded Perturbation of $A$}

Here we consider a specific bounded perturbation of the generator $A$ given by (4.2). To this end let $\phi$, defined by (4.1), be such that $1 / \phi$ belongs to $L^{1}\left(\mathbb{R}_{+}\right)$. Then, compare Sect. 3.2 in [3],

$$
B f(x):=\frac{1}{\phi(x)} \int_{0}^{+\infty} c(y) \phi(y) f(y) \mathrm{d} y, \quad x \in \mathbb{R}_{+}, f \in L^{1}\left(\mathbb{R}_{+}\right),
$$

where $c$ is a continuous and compactly supported nonnegative function on $\mathbb{R}_{+}$ satisfying $\|c\|_{1}=1$, defines a bounded operator in $L^{1}\left(\mathbb{R}_{+}\right)$with

$$
\|B\|_{1}=\|1 / \phi\|_{1}\|c \phi\|_{\infty} .
$$

Note that $B^{2}=B$, since

$$
B^{2} f=\frac{1}{\phi} \int_{0}^{+\infty} c(y) \phi(y) B f(y) \mathrm{d} y=B f \int_{0}^{+\infty} c(y) \mathrm{d} y=B f\|c\|_{1}=B f
$$

for all $f \in L^{1}\left(\mathbb{R}_{+}\right)$. This implies that $\|B\|_{1} \geq 1$ and $\mathrm{e}^{t B}=\left(\mathrm{e}^{t}-1\right) B+I$ for all $t \geq 0$, where $I$ is the identity operator in $L^{1}\left(\mathbb{R}_{+}\right)$. Consequently, since $B$ is nonnegative,

$$
\left\|\mathrm{e}^{t B}\right\|_{1}=\left(\mathrm{e}^{t}-1\right)\|B\|_{1}+1, \quad t \geq 0 .
$$

For each $\omega>0$ we denote

$$
B_{\omega}:=B-(1+\omega) I,
$$

and ask for which $\omega$ the semigroup generated by $A+B_{\omega}$ is uniformly exponentially stable.

Example 4.2. As a particular example of the above setup we let $\psi(x):=1$, or equivalently $\phi(x):=\mathrm{e}^{x}$, for all $x \in \mathbb{R}_{+}$. Then, see (4.2), $A f=f^{\prime}+f$ for each $f \in \mathcal{D}(A)=\left\{f \in L^{1}\left(\mathbb{R}_{+}\right): f^{\prime} \in L^{1}\left(\mathbb{R}_{+}\right)\right\}$, and $\left\|\mathrm{e}^{t A}\right\|_{1}=M_{t}=\mathrm{e}^{t}$ for every $t \geq 0$.

First we use the Dyson-Phillips estimate. If we write $A+B_{\omega}=[A-(1+$ $\omega) I]+B$, then by (3.3) with $A$ replaced by $A-(1+\omega) I$ we obtain

$$
\left\|\mathrm{e}^{t\left(A+B_{\omega}\right)}\right\|_{1} \leq \mathrm{e}^{\left(-\omega+\|B\|_{1}\right) t}, \quad t \geq 0 .
$$

This implies that the semigroup generated by $A+B_{\omega}$ is uniformly exponentially stable for every

$$
\omega>\|B\|_{1} .
$$

On the other hand, in order to apply Gil's theorem to $A+B_{\omega}$, we assume additionally (for simplicity) that $c$ is continuously differentiable and $c(0)=0$. By (4.4) and (4.7), the integral $\mathcal{J}_{\omega}:=\int_{0}^{+\infty}\left\|\mathrm{e}^{t A}\right\|_{1}\left\|\mathrm{e}^{t B_{\omega}}\right\|_{1} \mathrm{~d} t$ converges if and only if $\omega>1$. Furthermore, for each $f \in L^{1}\left(\mathbb{R}_{+}\right)$we have $(\phi B f)^{\prime} / \phi=0$, 
hence $\mathcal{D}(A)$ is invariant for $B$. Also, for every $f \in \mathcal{D}(A)$ it follows that $A B f=$ $-B f+B f=0$ and, by integration by parts,

$$
B A f=\frac{1}{\phi} \int_{0}^{+\infty}\left[c(y) \phi(y)-(c(y) \phi(y))^{\prime}\right] f(y) \mathrm{d} y ;
$$

here we used the fact that $c$ is differentiable, compactly supported and that $c(0)=0$. This shows that $A B-B A$ has a bounded extension $K$ to $L^{1}\left(\mathbb{R}_{+}\right)$ and

$$
\|K\|_{1}=\left\|c \phi-(c \phi)^{\prime}\right\|_{\infty}=\left\|c^{\prime} \phi\right\|_{\infty} .
$$

By Gil's theorem, for every $\omega>1$ the semigroup generated by $A+B_{\omega}$ is uniformly exponentially stable, provided that

$$
\|K\|_{1}^{\frac{1}{2}} \mathcal{J}_{\omega}=\frac{\|K\|_{1}^{\frac{1}{2}}}{\omega}\left(1+\frac{\|B\|_{1}}{\omega-1}\right)<1,
$$

or, equivalently, that

$$
\omega>\omega_{\kappa}:=\frac{1}{2}\left(1+\kappa+\sqrt{(\kappa-1)^{2}+4 \kappa\|B\|_{1}}\right),
$$

holds with $\kappa=\|K\|_{1}^{1 / 2}$.

Remark 4.3. We showed in Example 4.2 that the semigroup generated by $A+$ $B_{\omega}$ is uniformly exponentially stable, provided that

$$
\omega>\|B\|_{1} \quad \text { or } \quad \omega>\omega_{\|K\|_{1}^{1 / 2}}
$$

where the first estimate is obtained via the Dyson-Phillips formula, and the second one via Gil's theorem. To compare both approaches, or, in other words, to compare $\|B\|_{1}$ and $\omega_{\|K\|_{1}^{1 / 2}}$ we should somehow express $\|K\|_{1}$ in terms of $\|B\|_{1}$, and this is not possible if we do not know $c$. However, we can make some estimates. Because $c \phi$ is continuous and compactly supported, we have, see (4.6), $\|B\|_{1}=\|c \phi\|_{\infty}=c\left(x_{0}\right) \phi\left(x_{0}\right)$ for an $x_{0} \in[0,+\infty)$. Assuming that $c$ is differentiable, which we did when we used Gil's theorem, it follows that $(c \phi)^{\prime}\left(x_{0}\right)=0$, and consequently

$$
\|K\|_{1} \geq\|B\|_{1}
$$

by (4.8). Hence, if $\omega>\omega_{\|K\|_{1}^{1 / 2}}$, then in particular

$$
\omega>\omega_{\|K\|_{1}^{1 / 2}} \geq \omega_{\|B\|_{1}^{1 / 2}}
$$

for $[1,+\infty) \ni \kappa \mapsto \omega_{\kappa}$ is increasing. The inequality $\omega_{\|B\|_{1}^{1 / 2}}>\|B\|_{1}$ holds if and only if $\|B\|_{1}<\beta$, where $\beta$ is approximately 5.05. In other words, if $\|B\|_{1}<\beta$, then the Dyson-Phillips approach provides a better estimate on when the semigroup generated by $A+B_{\omega}$ is uniformly exponentially stable. On the other hand, if $\|B\|_{1}>\beta$, then the estimate obtained by Gil's theorem may be better. However, in this case it depends on how much bigger $\|K\|_{1}$ is from $\|B\|_{1}$, and this depends on the choice of the function $c$. If, for example, $c$ 
is chosen so that $\|K\|_{1} \geq\|B\|_{1}^{2}$, then the Dyson-Phillips estimate gives better results no matter how big $\|B\|_{1}$ is. Finally, we also stress that similar analysis can be made for Gil's theorem with Bobrowski's improvement, however, the calculations are more involved here.

It is one of the advantages of the Dyson-Phillips approach that we do not need to calculate $\mathcal{J}$ or $\|K\|$ explicitly, which is not easy in general, as we see in the following example.

Example 4.4. We consider the same operators $A$ and $B$ as in Example 4.2, however, we redefine the function $\psi$ as $\psi(x):=(1-\gamma) x^{-\gamma}$ for all $x>0$ (the value of $\psi$ at 0 does not matter) and fixed $\gamma \in(0,1)$. Then

$$
\phi(x)=\mathrm{e}^{x^{1-\gamma}}, \quad x \in \mathbb{R}_{+},
$$

and for $A$ defined by (4.2) we have

$$
\left\|\mathrm{e}^{t A}\right\|_{1}=M_{t}=\sup _{x \in \mathbb{R}_{+}} \mathrm{e}^{(x+t)^{1-\gamma}-x^{1-\gamma}}=\mathrm{e}^{t^{1-\gamma}}, \quad t \geq 0 .
$$

As before, by the Dyson-Phillips estimate, see (3.3), the semigroup generated by $A+B_{\omega}=A+B-(1+\omega) I$ is uniformly exponentially stable, provided that $\omega>\|B\|_{1}$. On the other hand, in order to apply Gil's theorem, for $\omega>0$ we calculate

$$
\begin{aligned}
\mathcal{J}_{\omega} & =\int_{0}^{+\infty} \mathrm{e}^{-(1+\omega) t}\left\|\mathrm{e}^{t A}\right\|_{1}\left\|\mathrm{e}^{t B}\right\|_{1} \mathrm{~d} t \\
& =\int_{0}^{+\infty} \mathrm{e}^{-\omega t} \mathrm{e}^{t^{1-\gamma}}\left[\left(1-\mathrm{e}^{-t}\right)\|B\|_{1}+\mathrm{e}^{-t}\right] \mathrm{d} t \\
& =\sum_{n=0}^{+\infty} \frac{1}{n !} \int_{0}^{+\infty} t^{(1-\gamma) n} \mathrm{e}^{-\omega t}\left[\left(1-\mathrm{e}^{-t}\right)\|B\|_{1}+\mathrm{e}^{-t}\right] \mathrm{d} t \\
& =\sum_{n=0}^{+\infty} \frac{\Gamma((1-\gamma) n+1)}{n !}\left[\frac{\|B\|_{1}}{\omega^{(1-\gamma) n+1}}+\frac{1-\|B\|_{1}}{(1+\omega)^{(1-\gamma) n+1}}\right],
\end{aligned}
$$

where $\Gamma$ is the Gamma function. Of course $\mathcal{J}_{\omega}$ converges for all $\omega>0$, since $\int_{0}^{+\infty} \mathrm{e}^{-\omega t} \mathrm{e}^{t^{1-\gamma}} \mathrm{d} t$ does so, however, even if we know $\|B\|_{1}$, it is really difficult to check, when condition (c) of Gil's theorem holds.

\section{Abstract Setup}

We consider the examples discussed in Sect. 4.1 as a special case of a general setup. We prove that in this setup it is possible to find an explicit formula for perturbed semigroups using the Dyson-Phillips theorem, which allows us to find a simple sufficient condition for the uniform exponential stability. Additionally, it turns out that in this setup the condition follows from condition (a) of Gil's theorem. 
Let $A$ be a generator of a strongly continuous semigroup in a Banach lattice $X$ equipped with the norm $\|\cdot\|$. Moreover, let $B$ be a bounded nonnegative operator in $X$ such that

$$
B^{2}=B \quad \text { and } \quad \mathrm{e}^{t A} B=B, t \geq 0 .
$$

As in Sect. 4.1, we note that $\mathrm{e}^{t B}=\left(\mathrm{e}^{t}-1\right) B+I$, where $I$ is the identity operator in $X$, and that

$$
\left\|\mathrm{e}^{t B}\right\|=\left(\mathrm{e}^{t}-1\right)\|B\|+1
$$

holds for all $t \geq 0$. Notice also that the operator $B$ given by (4.5) satisfies (5.1). Indeed, we checked that $B^{2}=B$ in Sect. 4.1, and additionally for $A$ defined by (4.2) we have

$$
\mathrm{e}^{t A} B f=\frac{\tau_{t} \phi}{\phi} \tau_{t}(1 / \phi) \int_{0}^{+\infty} c(y) \phi(y) f(y) \mathrm{d} y=B f, \quad t \geq 0, f \in L^{1}\left(\mathbb{R}_{+}\right) .
$$

In the above setup we find an explicit formula for the perturbed semigroup generated by $A+B$ (see $[2,8.2 .23]$ and [1] for more examples in this fashion), and consequently we provide a sufficient condition for its uniform exponential stability.

Theorem 5.1. Let $A$ be a generator of a strongly continuous semigroup in a Banach lattice $X$, and let $B$ be a bounded nonnegative operator in $X$ satisfying (5.1). The semigroup generated by $A+B$ is given by

$$
\mathrm{e}^{t(A+B)}=\mathrm{e}^{t A}+B \int_{0}^{t} \mathrm{e}^{t-s} \mathrm{e}^{s A} \mathrm{~d} s, \quad t \geq 0 .
$$

Moreover, for every $\omega>0$ the semigroup generated by

$$
A+B_{\omega}:=A+B-(1+\omega) I
$$

is uniformly exponentially stable, provided that

$$
\int_{0}^{+\infty} \mathrm{e}^{-(1+\omega) t}\left\|\mathrm{e}^{t A}\right\| \mathrm{d} t<+\infty .
$$

Proof. By the Dyson-Phillips formula, see (3.1), for all $t \geq 0$ the operator $\mathrm{e}^{t(A+B)}$ is given by the series $\sum_{n=0}^{+\infty} T_{n}(t)$, where $T_{0}(t)=\mathrm{e}^{t A}$, and

$$
T_{n+1}(t)=B \int_{0}^{t} T_{n}(s) \mathrm{d} s, \quad n \geq 0, t \geq 0 .
$$

Hence, it is easy to check by induction that

$$
T_{n+1}(t)=\frac{B}{n !} \int_{0}^{t}(t-s)^{n} \mathrm{e}^{s A} \mathrm{~d} s, \quad n \geq 0, t \geq 0 .
$$

Indeed, this holds for $n=0$, and if we assume that $T_{n+1}(t)$ is given by the above formula, then, by (5.5) and the fact that $B^{2}=B$,

$$
T_{n+2}(t)=\frac{B}{n !} \int_{0}^{t} \mathrm{e}^{u A} \int_{u}^{t}(s-u)^{n} \mathrm{~d} s \mathrm{~d} u=\frac{B}{(n+1) !} \int_{0}^{t}(t-u)^{n+1} \mathrm{e}^{u A} \mathrm{~d} u .
$$


Therefore, for each $t \geq 0$,

$$
\mathrm{e}^{t(A+B)}=\sum_{n=0}^{+\infty} T_{n}(t)=\mathrm{e}^{t A}+B \int_{0}^{t} \mathrm{e}^{s A} \sum_{n=0}^{+\infty} \frac{(t-s)^{n}}{n !} \mathrm{d} s,
$$

which implies (5.3). Consequently

$$
\left\|\mathrm{e}^{t\left(A+B_{\omega}\right)}\right\| \leq \mathrm{e}^{-(1+\omega) t}\left\|\mathrm{e}^{t A}\right\|+\|B\| \mathrm{e}^{-\omega t} \int_{0}^{t} \mathrm{e}^{-s}\left\|\mathrm{e}^{s A}\right\| \mathrm{d} s, \quad t \geq 0 .
$$

Assuming that $C:=\int_{0}^{+\infty} \mathrm{e}^{-(1+\omega) t}\left\|\mathrm{e}^{t A}\right\| \mathrm{d} t$ is finite, we have

$$
\int_{0}^{+\infty}\left\|\mathrm{e}^{t\left(A+B_{\omega}\right)}\right\| \mathrm{d} t \leq C+\|B\| \int_{0}^{+\infty}\left\|\mathrm{e}^{s A}\right\| \int_{s}^{+\infty} \mathrm{e}^{-s-\omega t} \mathrm{~d} t \mathrm{~d} s=\frac{\omega+\|B\|}{\omega} C,
$$

which completes the proof.

We stress that condition (5.4) does not depend on the operator B. Moreover, in the context of Gil's theorem, condition (5.4) is significantly weaker than conditions (a)-(d). More specifically, the following result holds.

Proposition 5.2. Suppose, under the assumptions of Theorem 5.1, that condition (a) of Gil's theorem is satisfied with $B$ replaced by $B_{\omega}=B-(1+\omega) I$ for an $\omega>0$, that is the integral $\int_{0}^{+\infty}\left\|\mathrm{e}^{t A}\right\|\left\|\mathrm{e}^{t B_{\omega}}\right\| \mathrm{d} t$ converges. Then condition (5.4) holds, and the semigroup generated by $A+B_{\omega}$ is uniformly exponentially stable.

Proof. By (5.2) we have

$$
\left\|\mathrm{e}^{t A}\right\|\left\|\mathrm{e}^{t B_{\omega}}\right\|=\mathrm{e}^{-\omega t}\left\|\mathrm{e}^{t A}\right\|\left[\left(1-\mathrm{e}^{-t}\right)\|B\|+\mathrm{e}^{-t}\right], \quad t \geq 0 .
$$

Since the right-hand side is of order $\mathrm{e}^{-\omega t}\left\|\mathrm{e}^{t A}\right\|$ as $t \rightarrow+\infty$, the result follows.

As a simple consequence of Theorem 5.1 we provide a much better estimate on when the semigroups generated by the operators $A+B_{\omega}$ considered in Examples 4.2 and 4.4 are uniformly exponentially stable.

Example 5.3. Let $A$ and $B$ be as in Example 4.2 or 4.4. Then for all $t \geq 0$, $\left\|\mathrm{e}^{t A}\right\|_{1}=M_{t}$, where $M_{t}=\mathrm{e}^{t}$ or $M_{t}=\mathrm{e}^{t^{1-\gamma}}$, respectively. Hence,

$$
\int_{0}^{+\infty} \mathrm{e}^{-(1+\omega) t}\left\|\mathrm{e}^{t A}\right\| \mathrm{d} t
$$

converges for all $\omega>0$. Therefore, for such $\omega$, the semigroup $\left\{\mathrm{e}^{t\left(A+B_{\omega}\right)}\right\}_{t \geq 0}$ is uniformly exponentially stable by Theorem 5.1 .

Finally, Theorem 5.1 applies also to the example considered by Bobrowski in [3]. We prove Proposition 1 from the cited paper. 
Example 5.4. Let $X=C[0,1]$ be the space of continuous functions defined on $[0,1]$. The operator $A$ in $X$ given by

$$
A f(0)=0 \quad \text { and } \quad A f(x)=\frac{-x}{1+x}[(1+x) f(x)]^{\prime}, \quad x \in(0,1]
$$

for all $f$ in the domain $\mathcal{D}(A)$ composed of all functions $f \in C[0,1]$ such that $x \mapsto(1+x) f(x)$ is differentiable on $(0,1]$ and satisfying $\lim _{x \rightarrow 0^{+}} x[(1+$ $x) f(x)]^{\prime}=0$, generates the strongly continuous semigroup

$$
\mathrm{e}^{t A} f(x)=\frac{1+x \mathrm{e}^{-t}}{1+x} f\left(x \mathrm{e}^{-t}\right), \quad t \geq 0, x \in[0,1], f \in C[0,1] .
$$

We define the operator $B$ in $C[0,1]$ by

$$
B f(x)=\frac{1}{1+x} \int_{0}^{1} c(y)(1+y) f(y) \mathrm{d} y, \quad x \in[0,1], f \in C[0,1],
$$

where $c$ is a nonnegative continuous function on [0,1] satisfying $\int_{0}^{1} c(y) \mathrm{d} y=1$. We easily check that $B$ is bounded and that $B^{2}=B$. Moreover, $\mathrm{e}^{t A} B=B$ for all $t \geq 0$. By Theorem 5.1 it follows that for all $\omega>0$ the semigroup generated by $A+B_{\omega}=A+B-(1+\omega) I$, where $I$ is the identity operator in $C[0,1]$, is uniformly exponentially stable.

\section{Compliance with Ethical Standards}

Conflict of interest The author declare that they have no conflict of interest.

Open Access. This article is licensed under a Creative Commons Attribution 4.0 International License, which permits use, sharing, adaptation, distribution and reproduction in any medium or format, as long as you give appropriate credit to the original author(s) and the source, provide a link to the Creative Commons licence, and indicate if changes were made. The images or other third party material in this article are included in the article's Creative Commons licence, unless indicated otherwise in a credit line to the material. If material is not included in the article's Creative Commons licence and your intended use is not permitted by statutory regulation or exceeds the permitted use, you will need to obtain permission directly from the copyright holder. To view a copy of this licence, visit http://creativecommons. org/licenses/by/4.0/.

\section{Appendix: Proof of Lemma 4.1}

The argument is similar to the standard proof for the left translations semigroup, see for example [9, p. 9-10]. However, because the function $\phi$ is not necessarily bounded, we have to be a bit careful. The main difference lies in formula (5.7). On the right-hand side there is the additional term $I(x, t)$, which 
is not present in formula $(*)$ in $[9$, p. 10]. Notice also that if we denote the right-hand side of $(4.3)$ by $T(t) f$, then

$$
T(t)=J^{-1} \tau_{t} J, \quad t \geq 0,
$$

where $\left\{\tau_{t}\right\}_{t>0}$ is the semigroup of left translations in $L^{1}\left(\mathbb{R}_{+}\right)$, and (formally) $J f:=\phi f, J^{-1} f:=f / \phi$. However, for unbounded $\phi$ the operator $J$ is not an operator in $L^{1}\left(\mathbb{R}_{+}\right)$, and we cannot claim that $\{T(t)\}_{t \geq 0}$ is similar, see $[5$, II.2.1], to the semigroup $\left\{\tau_{t}\right\}_{t \geq 0}$.

Proof of Lemma 4.1. Clearly $T(0)$ is the identity operator in $L^{1}\left(\mathbb{R}_{+}\right)$and $T(s+t)=T(s) T(t)$ for all $s, t \geq 0$. Moreover, for each $f \in L^{1}\left(\mathbb{R}_{+}\right)$we have

$$
\|T(t) f-f\|_{1} \leq \int_{0}^{+\infty} m_{t}\left|\tau_{t} f-f\right|+\int_{0}^{+\infty}\left|m_{t}-1\right| f, \quad t \geq 0,
$$

where $m_{t}:=\tau_{t} \phi / \phi$. Note that $\sup _{t \in[0,1]}\left\|m_{t}\right\|_{\infty}=\sup _{t \in[0,1]} M_{t}$ is finite, since $t \mapsto M_{t}$ is continuous. Additionally, $m_{t}(x)$ converges to 1 as $t \rightarrow 0^{+}$for every $x \in \mathbb{R}_{+}$. Hence, by the continuity of translations in $L^{1}\left(\mathbb{R}_{+}\right)$and the Lebesgue Dominated Convergence Theorem, we obtain that $T(t) f$ converges to $f$ in $L^{1}\left(\mathbb{R}_{+}\right)$as $t \rightarrow 0^{+}$, proving that $\{T(t)\}_{t \geq 0}$ is a strongly continuous semigroup. We have $\|T(t)\|_{1} \leq M_{t}, t \geq 0$, and in order to show that $\|T(t)\|_{1}$ equals $M_{t}$ observe that, since $x \mapsto m_{t}(x)$ is continuous for every $t \geq 0$,

$$
M_{t}=m_{t}\left(x_{0}\right) \text { for some } x_{0} \in \mathbb{R}_{+} \quad \text { or } \quad M_{t}=\limsup _{x \rightarrow+\infty} m_{t}(x) \text {. }
$$

In the first case we take $f_{n}, n \geq 1$ to be the characteristic function of the interval $\left[x_{0}, x_{0}+1 / n\right)$. Clearly $\left\|n f_{n}\right\|_{1}=1$ and

$$
\lim _{n \rightarrow+\infty} n\left\|T(t) f_{n}\right\|_{1}=\lim _{n \rightarrow+\infty} n \int_{0}^{1 / n} m_{t}\left(x_{0}+x\right) \mathrm{d} x=m_{t}\left(x_{0}\right),
$$

which proves the claim. In the second case the argument is similar.

We are left with proving that $A$ is the generator of $\{T(t)\}_{t \geq 0}$. To this end let $f$ belong to $\mathcal{D}(A)$ and for $t \geq 0$ denote $T_{t} f:=t^{-1}(T(t) f-f)$. Then for all $t \geq 0$ and $x \in \mathbb{R}_{+}$we have

$$
\begin{aligned}
T_{t} f(x) & =\frac{1}{\phi(x)} t^{-1} \int_{0}^{t}[\phi(x+y) f(x+y)]^{\prime} \mathrm{d} y \\
& =\frac{1}{\phi(x)} \int_{0}^{1}[\phi(x+y t) f(x+y t)]^{\prime} \mathrm{d} y .
\end{aligned}
$$

Hence, by (4.2) and the Fubini Theorem,

$$
\left\|T_{t} f-A f\right\|_{1} \leq \int_{0}^{1} \int_{0}^{+\infty} \frac{1}{\phi(x)}\left|[\phi(x+y t) f(x+y t)]^{\prime}-[\phi(x) f(x)]^{\prime}\right| \mathrm{d} x \mathrm{~d} y .
$$

Because $f \in \mathcal{D}(A)$, for all $t, y \in[0,1]$ the inner integral is bounded by

$$
\left\|m_{y t} \cdot \tau_{y t}\left((\phi f)^{\prime} / \phi\right)\right\|_{1}+\left\|(\phi f)^{\prime} / \phi\right\|_{1} \leq\left(1+\sup _{t \in[0,1]} M_{t}\right)\left\|(\phi f)^{\prime} / \phi\right\|_{1} .
$$


Hence, by the Lebesgue Dominated Convergence Theorem and by the continuity of translations in $L^{1}\left(\mathbb{R}_{+}\right)$, compare (5.6), $T_{t} f$ converges to $A f$ in $L^{1}\left(\mathbb{R}_{+}\right)$ as $t \rightarrow 0^{+}$.

On the other hand, assume that for some $f \in L^{1}\left(\mathbb{R}_{+}\right), T_{t} f$ converges to $g \in L^{1}\left(\mathbb{R}_{+}\right)$as $t \rightarrow 0^{+}$. We easily check that for every $t \geq 0$ and $x \in \mathbb{R}_{+}$,

$$
\int_{0}^{x} T_{t} f(y) \mathrm{d} y=t^{-1}\left[\int_{x}^{x+t} f(y) \mathrm{d} y-\int_{0}^{t} f(y) \mathrm{d} y+I(x, t)\right],
$$

where

$$
I(x, t):=\int_{t}^{x+t} \phi(y) f(y)\left[\frac{1}{\phi(y-t)}-\frac{1}{\phi(y)}\right] \mathrm{d} y, \quad t \geq 0, x \in \mathbb{R}_{+} .
$$

For all $t$ and $x$ as above we have

$$
\left|\frac{1}{\phi(y-t)}-\frac{1}{\phi(y)}\right| \leq 2 \exp \left(\int_{0}^{x+t}\left|\psi\left(y^{\prime}\right)\right| \mathrm{d} y^{\prime}\right), \quad y \in(t, x+t) .
$$

For every $x \in \mathbb{R}_{+}$the right-hand side is bounded as a function of $t \in[0,1]$, thus, by the Lebesgue Dominated Convergence Theorem, we obtain

$$
\lim _{t \rightarrow 0^{+}} t^{-1} I(x, t)=\int_{0}^{x} \psi(y) f(y) \mathrm{d} y, \quad x \in \mathbb{R}_{+} .
$$

Consequently, the right-hand side of (5.7) converges for almost every $x$ to $f(x)-f(0)+\int_{0}^{x} \psi(y) f(y) \mathrm{d} y$ as $t \rightarrow 0^{+}$. However, by assumption, the lefthand side of (5.7) converges to $\int_{0}^{x} g(y) \mathrm{d} y$ for all $x \in \mathbb{R}_{+}$. Therefore, $f$ is absolutely continuous and $f^{\prime}+\psi f=g$, which completes the proof.

\section{References}

[1] Bobrowski, A.: Quasi-stationary distributions of a pair of Markov chains related to time evolution of a DNA locus. Adv. Appl. Probab. 36(1), 57-77 (2004)

[2] Bobrowski, A.: Functional Analysis for Probability and Stochastic Processes. Cambridge University Press, Cambridge (2005)

[3] Bobrowski, A.: A note on integrability of the norm of a perturbed semigroup. Results Math. 75(3), 105-8 (2020)

[4] Brendle, S.: On the asymptotic behavior of perturbed strongly continuous semigroups. Math. Nachr. 226, 35-47 (2001)

[5] Engel, K.-J., Nagel, R.: One-Parameter Semigroups for Linear Evolution Equations. Springer-Verlag, New York (2000)

[6] Gil', M.: Semigroups of sums of two operators with small commutators. Semigr. Forum 98(1), 22-30 (2019)

[7] Gil', M.: Norm estimates for a semigroup generated by the sum of two operators with an unbounded commutator. Results Math. 751, 10 (2020)

[8] Meyer, C.: Matrix Analysis and Applied Linear Algebra. Society for Industrial and Applied Mathematics (SIAM), Philadelphia, PA (2000) 
[9] Nagel, R. (ed.): One-parameter Semigroups of Positive Operators. Springer, Cham (1986)

[10] Preda, C.: A survey on perturbed exponentially stable $C_{0}$-semigroups. Qual. Theory Dyn. Syst. 15(2), 541-551 (2016)

[11] Stampfli, J.: The norm of a derivation. Pac. J. Math. 33, 737-747 (1970)

Adam Gregosiewicz

Lublin University of Technology

ul. Nadbystrzycka $38 \mathrm{~A}$

20-618 Lublin

Poland

e-mail: a.gregosiewicz@pollub.pl

Received: December 9, 2020.

Accepted: January 27, 2021.

Publisher's Note Springer Nature remains neutral with regard to jurisdictional claims in published maps and institutional affiliations. 